\title{
Growing the Digital Repository of Mathematical Formulae with Generic $\mathrm{HT}_{\mathrm{E}} \mathrm{X}$ Sources *
}

\author{
Howard S. Cohl ${ }^{1}$, Moritz Schubotz ${ }^{2}$, Marjorie A. McClain ${ }^{1}$, Bonita \\ V. Saunders ${ }^{1}$, Cherry Y. Zou ${ }^{3}$, Azeem S. Mohammed ${ }^{3}$, and Alex A. Danoff ${ }^{4}$ \\ 1 Applied and Computational Mathematics Division, National Institute of Standards \\ and Technology (NIST), Gaithersburg, Maryland, U.S.A., \\ \{howard.cohl, marjorie.mcclain, bonita.saunders\}@nist.gov \\ 2 Database Systems and Information Management Group, Technische Universität, \\ Berlin, Germany, schubotz@tu-berlin.de \\ 3 Poolesville High School, Poolesville, Maryland, U.S.A., \\ $\{$ chzou2009, azeemsm\}@gmail.com \\ 4 Wootton High School, Rockville, Maryland, U.S.A. aadanoff@gmail.com
}

\begin{abstract}
One initial goal for the DRMF is to seed our digital compendium with fundamental orthogonal polynomial formulae. We had used the data from the NIST Digital Library of Mathematical Functions (DLMF) as initial seed for our DRMF project. The DLMF input LATEX source already contains some semantic information encoded using a highly customized set of semantic $\mathrm{LT}_{\mathrm{E}} \mathrm{X}$ macros. Those macros could be converted to content MAThML using IATEXML. During that conversion the semantics were translated to an implicit DLMF content dictionary. This year, we have developed a semantic enrichment process whose goal is to infer semantic information from generic $\mathrm{L}_{\mathrm{A}} \mathrm{T} \mathrm{X}$ sources. The generated context-free semantic information is used to build DRMF formula home pages for each individual formula. We demonstrate this process using selected chapters from the book "Hypergeometric Orthogonal Polynomials and their $q$-Analogues" (2010) by Koekoek, Lesky and Swarttouw (KLS) as well as an actively maintained addendum to this book by Koornwinder (KLSadd). The generic input KLS and KLSadd IATEX sources describe the printed representation of the formulae, but does not contain explicit semantic information. See http://drmf .wmflabs.org.
\end{abstract}

\section{Introduction}

Formula home pages are the principal conceptual objects for the DRMF project. These should contain the full context-free semantic information concerning individual orthogonal polynomial and special function (OPSF) formulae. The DRMF is designed for a mathematically literate audience and should (1) facilitate interaction among a community of mathematicians and scientists interested in compendia formulae data for orthogonal polynomials and special functions; (2) be expandable, allowing the input of new formulae from the literature; (3) represent the context-free full semantic information concerning individual formulae; (4) have a user friendly, consistent, and hyperlinkable viewpoint and authoring perspective; (5) contain easily searchable mathematics; and (6) take advantage

\footnotetext{
* The final publication is available at http://link.springer.com.
} 


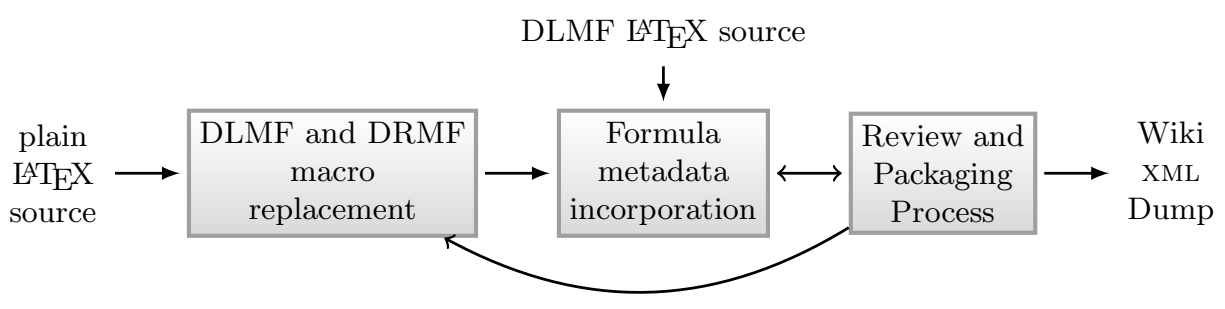

Fig. 1. Data flow of seeding projects. For most of the input $\mathrm{LT}_{\mathrm{E}} \mathrm{X}$ source distributions, DLMF and DRMF macros are not incorporated. For the DLMF IATEX source, the DLMF macros are already incorporated.

of modern MATHML tools for easy-to-read, scalably rendered content driven mathematics. In this paper we will discuss the DRMF seeding projects whose goal is to import data, for example, from traditional print media (cf. Figure 1).

We are investigating various sources for seed material in the DRMF [3]. We have been given permission to use a variety of input resources to generate our online compendium of mathematical formulae. The current sources that we are incorporating into the DRMF are given as follows: (1) NIST Digital Library of Mathematical Functions (DLMF ${ }^{1}$ ) [1, 6]; (2) Chapters 1, 9, and 14 (a total of 228 pages with about 1800 formulae) from the Springer-Verlag book "Hypergeometric Orthogonal Polynomials and their q-Analogues" (2010) by Koekoek, Lesky and Swarttouw (KLS) [7]; (3) Tom Koornwinder's Additions to the formula lists in "Hypergeometric orthogonal polynomials and their q-Analogues" by Koekoek, Lesky and Swarttouw (KLSadd) [10]; (4) Wolfram Computational Knowledge of Continued Fractions Project (eCF); and the Bateman Manuscript Project (BMP) $[4,5]$ (see Table 1). Note that the DLMF, KLS, KLSadd, and eCF datasets are currently being processed within our pipeline. For the BMP dataset, we have furnished high-quality print scans to Alan Sexton and are currently waiting on the math OCR generated $\mathrm{AT}_{\mathrm{E}} \mathrm{X}$ output for this dataset which is currently being generated. In this paper we focus on DRMF seeding of generic $\mathrm{LAT}_{\mathrm{E}} \mathrm{X}$ sources, namely those which do not contain explicit semantic information.

\section{Seeding with Generic $\mathrm{H}_{\mathrm{E}} \mathrm{X}$ X Sources}

DRMF seeding projects collect and stream OPSF mathematical formulae into formula pages. Formula pages are classified into those which list formulae in a broad category, and the individual formula home pages for each formula. Generated formula home pages are required to contain bibliographic information and usually contain a list of symbols, substitutions and constraints required by the formulae, proofs and formula names if available, as well as related notes. Every semantic formula entity (e.g., function, polynomial, sequence, operator, constant or set) has a unique name and a link to its definition or description.

\footnotetext{
${ }^{1}$ We use the typewriter font in this document to refer to our seeding datasets.
} 
Table 1. Overview of the first three stages of the DRMF project. Note that the numbers which are given are rough estimates.

\begin{tabular}{|c|c|c|c|}
\hline & Stage 1 & Stage 2 & Stage 3 \\
\hline StARTED IN & 2013 & 2014 & 2015 \\
\hline DATASET & $\begin{array}{c}\text { DLMF, } \\
\text { semantic LATEX }\end{array}$ & $\begin{array}{c}\text { KLS, } \\
\text { plain } \mathrm{ETT}_{\mathrm{E}} \mathrm{X}\end{array}$ & $\begin{array}{l}\text { eCF: Mathematica } \\
\text { BMP: book images }\end{array}$ \\
\hline $\begin{array}{l}\text { SEMANTIC } \\
\text { ENRICHMENT }\end{array}$ & $\begin{array}{c}\text { identify constraints, } \\
\text { substitutions, } \\
\text { notes, names, } \\
\text { proofs, ... }\end{array}$ & $\begin{array}{c}\text { add } \\
\text { new } \\
\text { semantic } \\
\text { macros }\end{array}$ & $\begin{array}{l}\text { image recognition } \\
\text { macro suggestion }\end{array}$ \\
\hline TEChnologies & $\begin{array}{c}\text { manual review, } \\
\text { rule-based } \\
\text { approaches }\end{array}$ & improved rules & $\begin{array}{c}\text { natural language } \\
\text { processing and } \\
\text { machine learning }\end{array}$ \\
\hline $\begin{array}{l}\text { NUMBER OF } \\
\text { FORMULA } \\
\text { HOME PAGES }\end{array}$ & 500 & 1500 & 5000 \\
\hline $\begin{array}{l}\text { HUMAN TIME } \\
\text { PER FORMULA } \\
\text { HOMEPAGE }\end{array}$ & 10 minutes & 5 minutes & 1 minute \\
\hline $\begin{array}{l}\text { TEST CORPORA } \\
\text { CONTRIBUTION }\end{array}$ & $\begin{array}{l}\text { gold standard } \\
\text { for constraint } \\
\text { and proof } \\
\text { detection }\end{array}$ & $\begin{array}{c}\text { gold standard } \\
\text { for } \\
\text { macro } \\
\text { replacement }\end{array}$ & $\begin{array}{l}\text { evaluation } \\
\text { metrics }\end{array}$ \\
\hline
\end{tabular}

For $\mathrm{AT}_{\mathrm{E}} \mathrm{X}$ sources which are extracted from the DLMF project, the semantic macros are already incorporated [11]. However, for generic sources such as the KLS dataset, the semantic macros need to be inserted in replacement for the ${ }_{\mathrm{A}} \mathrm{T}_{\mathrm{E}} \mathrm{X}$ source which represents that mathematical object.

Here we give representative examples for the trigonometric sine function, gamma function, Jacobi polynomial and little $q$-Laguerre/Wall polynomials, which are rendered respectively as $\sin z, \Gamma(z), P_{n}^{(\alpha, \beta)}(x)$, and $p_{n}(x ; a \mid q)$. These functions and orthogonal polynomials have $\mathrm{LAT}_{\mathrm{E}} \mathrm{X}$ presentations given respectively by $\backslash \sin z, \backslash \operatorname{Gamma}(z), P \_\wedge\{(\backslash$ alpha, $\backslash$ beta $)\}(x)$, and $p \_n(x ; a \mid q)$. The semantic representations for these functions and orthogonal polynomials are given respectively by $\backslash \sin @ @\{z\}, \backslash$ EulerGamma@ $\{z\}, \backslash J a c o b i\{\backslash$ alpha $\}\{\backslash$ beta $\}$ $\{\mathrm{n}\} @\{\mathrm{x}\}, \backslash$ littleqLaguerre $\{\mathrm{n}\} @\{\mathrm{x}\}\{\mathrm{a}\}\{\mathrm{q}\}$. The arguments before the @ or @@ symbols are parameters and the arguments after the@ or @@ symbol are in the domain of the functions and orthogonal polynomials. The different between the @ or @@ symbols indicates a specified difference in presentation, such as the inclusion of the parentheses or not in our trigonometric sine example. For the little $q$-Laguerre polynomials, one has three arguments within parentheses. These three arguments are separated by a semi-colon and a vertical bar. Our macro 
replacement algorithm indentifies these polynomials, and then extracts the information about what the contents of each argument is. Furthermore there are many ways in $\mathrm{IAT}_{\mathrm{E}} \mathrm{X}$ to represent open and close parenthese, our algorithm identifies these. Also, since the vertical bar in $\mathrm{HT}_{\mathrm{E}} \mathrm{X}$ can be represented by '"' or ' $\backslash$ mid', we search for both of these patterns. Our algorithm, for instance, also searches for and removes all $\mathrm{HAT}_{\mathrm{E} X}$ white-space characters such as those given by $\backslash, \backslash$ ! or $\backslash$ hspace \{\} . There are many other details about making our search and replace work, which we will not mention here.

\section{$3 \quad$ KLS Seeding Project}

In this section we describe how we augment the input KLS LATEX source in order to generate formula pages (see Figure 1). We are developing software processes input $\mathrm{IAT}_{\mathrm{E} X} \mathrm{X}$ source to generate output $\mathrm{H}_{\mathrm{E}} \mathrm{X}$ source with semantic mathematical macros incorporated. The semantic $\mathrm{H}_{\mathrm{E}} \mathrm{TX}$ macros that we are using (664 total with 147 currently being used for the DRMF project) are being developed by NIST for use in the DLMF and DRMF projects. Whenever possible, we use the standardized definitions from the NIST Digital Library of Mathematical Functions [6]. If the definitions are not available on the DLMF website, then we link to definition pages in the DRMF with included symbols lists. One main goal of this seeding project is to incorporate mathematical semantic information directly into the $\mathrm{AT}_{\mathrm{E} X} \mathrm{X}$ source. The advantage of incorporating this information directly into the $\mathrm{H}_{\mathrm{A}} \mathrm{T}_{\mathrm{E}} \mathrm{X}$ source is that mathematicians are capable of editing $\mathrm{L}_{\mathrm{A}} \mathrm{T}_{\mathrm{E}} \mathrm{X}$ whereas human editing of MATHML is not feasible. This enriched information can be further modified by mathematicians using their regular working environment.

For the 3 chapters of the KLS dataset plus the KLSadd dataset, a total number of 89 semantic macros were replaced a total of 3308 times. That's an average of 1.84 macros replaced per formula. Note that the KLSadd dataset is actively being maintained, and when a new version of it is published, in an automated fashion, incorporate this new information into the DRMF. This fraction will increase when more algebraic substitution formulae are included as formula metadata. The most common macro replacements are given as follows. The macro for the cosine function, Racah polynomial, Pochhammer symbol, $q$-hypergeometric function, Euler gamma function, and $q$-Pochhammer symbol were converted a total number of times equal to 117, 205, 237, 266, and 659. Our current conversions, which use a rule based approach, can be quite complicated due to the nature of the variety of combinations of $\mathrm{AT}_{\mathrm{E}} \mathrm{X}$ input for various OPSF objects. In $\mathrm{IAT}_{\mathrm{E}} \mathrm{X}$ there are many ways of representing parentheses which are usually used for function arguments. Also, there are many ways to represent spacing delimiters which can mostly be ignored as far as representing the common semantic information for a mathematical function call. Our software canonicalizes these additional meaningless degrees of freedom and generates easy-to-read semantic ${ }_{\mathrm{AT}} \mathrm{TX}$ source and improves the rendering. Developing automatic software which performs macro replacements for OPSF functions in $\mathrm{IAT}_{\mathrm{E}} \mathrm{X}$ is a challenging task. The current status of our rule-based approach is highly tailored to our specific KLS and KLSadd input $\mathrm{LT}_{\mathrm{E}} \mathrm{X}$ source. 
Historically, the desired need for formal consistency has driven mathematicians to adopt consistent and unique notations [2]. This is extremely beneficial in the long run. We have interacted on a regular basis with the authors of the KLS and KLSadd datasets. They agree that our assumptions about consistent notations are correct and they consider using our semantic $\mathrm{AT}_{\mathrm{E}} \mathrm{X}$ macros in future volumes. Certainly the benefit of using these macros in communicating with different computer systems is clear.

Once semantic macros are incorporated, the next task is to identify formula metadata. Formula metadata can be identified within and must be associated with formulae. One must then identify semantic information for the formula within the surrounding text to produce formula annotations which describe this semantic information. There are annotations which can be summarized as constraints, substitutions, proofs and formula names if available, as well as related notes. The automated extraction of formula metadata is a challenging aspect of the seeding project and future computer implementations might use machine learning methods to achieve this goal. However, we have built automated algorithms to extract formula metadata. We have for instance identified substitutions by associating definitions for algebraic or OPSF functions which are utilized in surrounding formulae. The automation process continues by merging these substitution formulae as annotations in the original formulae which use them. Another extraction algorithm we have developed is the identification of related variables, understanding their dependencies and merging corresponding annotations with the pre-existing formula metadata. We have manually reviewed the printed mathematics to identify formula metadata. After we have exhausted our current rule-based approach for extracting the formula annotations, we will perform the manual insertion of the missing identified annotations into the $\mathrm{HT}_{\mathrm{E} X}$ source. This will then be followed by careful checking and expert editorial review. This also evaluates the quality of our rule-based approach and creates a gold standard for future programs.

Once the formula metadata has been completely extracted from the text, then the remainder of the text should be removed and one is left with a list of $\mathrm{ITT}_{\mathrm{E}} \mathrm{X}$ formulae with associated metadata. From this list (at the current stage of our project), we use this semantic $\mathrm{IT}_{\mathrm{E}} \mathrm{X}$ source to generate Wikitext. One of the features of the generated Wikitext is that we use a glossary that we have developed of our DLMF and DRMF macros to identify semantic macros within a formula and its associated metadata. Presentation and meaningful content MATHML is generated from the DLMF and DRMF macros using a customized ATEXML server (http://gw125.iu.xsede.org) hosted by the XSEDE project that includes all generated semantic macros. From this glossary, we generate symbols lists for each formula which uses recognized symbols. The generated Wikitext is converted to the MediaWiki XML-Dump format, which is then bulk imported to our wiki instance. Our DRMF Wiki has been optimized for MATHML-output. Because we are using Mathoid to render mathematical expressions [14], browsers without MAтHML-support can display DRMF formulae within MediaWiki. However, 
some MATHML-related features (such as copying parts of the MATHML output) are not available on these browsers.

At the moment, There are 1282 KLS and KLSadd wikitext pages. The current number of KLS and KLSadd formula home pages is 1219 and the percentage of non-empty symbols lists in formula home pages is given by 98.6 percent. This number will increase as we continue to merge substitution formulae into associated metadata and as we continue to expand our macro replacement effort. We have detected 208 substitutions which originally appeared as formulae. We inserted these in an automated fashion into 515 formulae. The goal of our learning is to obtain a mostly unambiguous content representation of the mathematical OPSF formulae which we use.

\section{Future outlook}

The next seeding projects which we will focus on are those which correspond to image and Mathematica inputs (see Table 1). We have been given permission from Caltech to use the BMP dataset within the DRMF. In the BMP dataset, the original source for data are printed pages of books. We are currently collaborating on the development of mathematical optical character recognition (OCR) software [15] for use in this project. We plan to utilize this math OCR software to generate $\mathrm{AAT}_{\mathrm{E} X} \mathrm{X}$ output which will be incorporated with the DLMF and DRMF semantic macros using our developed macro replacement software.

We are already developing for our next source, namely the incorporation of the Wolfram eCF dataset into the DRMF. We have been furnished the Mathematica source (also known as Wolfram language) for this dataset and we are currently developing software which translates in both directions from the Wolfram language to our semantic $\mathrm{LT}_{\mathrm{E}} \mathrm{X}$ source with DRMF and DLMF macros incorporated (cf. Table 1).

For the DLMF source, due to the hard efforts of the DLMF team for more than the past ten years, we already have semantic macros implemented, and all that remains is to extract the metadata from the text associated with formulae, removing the text after the content has been transferred, converting formulae information in tables to lists of distinct formulae, and generating formula home pages. We already have mostly achieved this for DLMF Chapter 25 on the Riemann Zeta function and are currently at work on Chapters 5 (gamma function), 15 (hypergeometric function), 16 (generalized hypergeometric functions), 17 ( $q$ hypergeometric and related functions) and 18 (orthogonal polynomials) which will ultimately be merged with the KLS and KLSadd datasets. Then we will continue to the remainder of the DLMF chapters.

Once semantic information has been inserted into the $\mathrm{IAT}_{\mathrm{E}} \mathrm{X}$ source, there is a huge number of possibilities on how this information can be used. Given that our datasets are collections of OPSF formulae, we plan on taking advantage of the incorporated semantic information as an exploratory tool for symbolic and numerical experiments. For instance, one may use this semantic content to translate to computer algebra system (CAS) computer languages such as those used by Mathematica, Maple or Sage. One could then use the translated formulae 
while taking advantage of any of the features available in those software packages. We should also mention that the DRMF seeding projects generate real content MатнML. This has been a huge problem for Mathematics Information Retrieval research for many years $[9,12]$. One major contribution of the DRMF seeding projects is that they offer quite reasonable content MATHML.

From a methodological point of view, we are going to develop evaluation metrics that measure the degree of semantic formula enrichment. These should be able to evaluate new approaches such as mathematical language processing [13] and/or machine learning approaches based on the created gold standard. Additionally, we are considering the use of sTeX [8], in order to simplify the definition of new macros. Eventually, we can also develop a heuristic which suggests new semantic macros based on statistical analysis.

\section{Acknowledgements $^{2}$}

We are indebted to Wikimedia Labs, the XSEDE project, Springer-Verlag, the California Institute of Technology, and Wolfram Research Inc. for their contributions and continued support. We would also like to thank Roelof Koekoek, Tom Koornwinder, Roberto Costas-Santos, Eric Weisstein, Dan Lozier, Alan Sexton, Bruce Miller, Abdou Youssef, Charles Clark, Volker Markl, George Andrews, Mourad Ismail, and Dmitry Karp for their advice, invaluable assistance, and support.

\section{References}

[1] NIST Digital Library of Mathematical Functions. http://dlmf .nist.gov, Release 1.0.9 of 2014-08-29. Online companion to [6].

[2] G. E. Andrews, R. Askey, and R. Roy. Special functions, volume 71 of Encyclopedia of Mathematics and its Applications. Cambridge University Press, Cambridge, 1999.

[3] H. S. Cohl, M. A. McClain, B. V. Saunders, M. Schubotz, and J. C. Williams. Digital Repository of Mathematical Formulae. In S. M. Watt, J. H. Davenport, A. P. Sexton, P. Sojka, J. Urban, editor, Intelligent Computer Mathematics, Lecture Notes in Artificial Intelligence 8543, pages 419422. Springer, 2014.

[4] A. Erdélyi, W. Magnus, F. Oberhettinger, and F. G. Tricomi. Tables of integral transforms. Vols. 1-2. McGraw-Hill Book Company, Inc., New YorkToronto-London, 1954.

\footnotetext{
${ }^{2}$ The mention of specific products, trademarks, or brand names is for purposes of identification only. Such mention is not to be interpreted in any way as an endorsement or certification of such products or brands by the National Institute of Standards and Technology, nor does it imply that the products so identified are necessarily the best available for the purpose. All trademarks mentioned herein belong to their respective owners.
} 
[5] A. Erdélyi, W. Magnus, F. Oberhettinger, and F. G. Tricomi. Higher transcendental functions. Vols. 1-3. Robert E. Krieger Publishing Co. Inc., Melbourne, Fla., 1981.

[6] F. W. J. Olver and D. W. Lozier and R. F. Boisvert and C. W. Clark, editor. NIST Handbook of Mathematical Functions. Cambridge University Press, New York, NY, 2010. Print companion to [1].

[7] R. Koekoek, P. A. Lesky, and R. F. Swarttouw. Hypergeometric orthogonal polynomials and their q-analogues. Springer Monographs in Mathematics. Springer-Verlag, Berlin, 2010. With a foreword by Tom H. Koornwinder.

[8] M. Kohlhase. Using LaTeX as a semantic markup format. Mathematics in Computer Science, 2(2):279-304, 2008.

[9] M. Kohlhase and I. Sucan. A search engine for mathematical formulae. In Jacques Calmet, Tetsuo Ida, and Dongming Wang, editors, Artificial Intelligence and Symbolic Computation, volume 4120 of Lecture Notes in Computer Science, pages 241-253. Springer Berlin Heidelberg, 2006.

[10] T. H. Koornwinder. Additions to the formula lists in "Hypergeometric orthogonal polynomials and their $q$-analogues" by Koekoek, Lesky and Swarttouw. arXiv:1401.0815v2, 2015.

[11] B. R. Miller and A. Youssef. Technical Aspects of the Digital Library of Mathematical Functions. Annals of Mathematics and Artificial Intelligence, 38(1-3):121-136, 2003.

[12] M.-Q. Nghiem, G. Y. Kristianto, G. Topić, and A. Aizawa. Which One Is Better: Presentation-Based or Content-Based Math Search? In S. M. Watt, J. H. Davenport, A. P. Sexton, P. Sojka, and J. Urban, editors, Intelligent Computer Mathematics, volume 8543 of Lecture Notes in Computer Science, pages 200-212. Springer International Publishing, 2014.

[13] R. Pagel and M. Schubotz. Mathematical Language Processing Project. In M. England, J. H. Davenport, A. Kohlhase, M. Kohlhase, P. Libbrecht, W. Neuper, P. Quaresma, A. P. Sexton, P. Sojka, J. Urban, and S. M. Watt, editors. Joint Proceedings of the MathUI, OpenMath and ThEdu Workshops and Work in Progress track at CICM co-located with Conferences on Intelligent Computer Mathematics (CICM 2014), Coimbra, Portugal, July 7-11, 2014, volume 1186 of CEUR Workshop Proceedings. http://CEUR-WS.org, 2014.

[14] M. Schubotz and G. Wicke. Mathoid: Robust, Scalable, Fast and Accessible Math Rendering for Wikipedia. In Stephen M. Watt, James H. Davenport, Alan P. Sexton, Petr Sojka, and Josef Urban, editors, Intelligent Computer Mathematics, volume 8543 of Lecture Notes in Computer Science, pages 224-235. Springer International Publishing, 2014.

[15] A. P. Sexton. Abramowitz and Stegun - a resource for mathematical document analysis. In Johan Jeuring, John Campbell, Jacques Carette, Gabriel Dos Reis, Petr Sojka, Makarius Wenzel, and Volker Sorge, editors, Conferences on Intelligent Computer Mathematics (CICM 2012), volume 7362 of Lecture Notes in Computer Science, pages 159-168. Springer Berlin/Heidelberg, 2012. 